\title{
Phenotypic methods for detection of metallobetalactamase in Meropenem resistant Pseudomonas aeruginosa and molecular detection of blaIMP \& blaVIM genes carrying strains
}

\author{
Charanjeev kaur ${ }^{1, *}$, Poonam Sharma ${ }^{2}$, Sarbjeet Sharma ${ }^{3}$ \\ ${ }^{1}$ Assistant Professor, ${ }^{2}$ Professor, ${ }^{3}$ Professor \& Head, Dept. of Microbiology, Sri Guru Ram Das Institute of Medical Sciences and \\ Research, Amritsar, Punjab, India \\ *Corresponding Author: \\ Email: drcharanjeev@gmail.com
}

\begin{abstract}
Introduction and Objectives: Pseudomonas aeruginosa is one of the major organisms responsible for nosocomial infections such as pneumonia, UTIs, surgical site infections, and bloodstream infections. ${ }^{1}$ High intrinsic and acquired resistance to many antimicrobial agents have allowed $P$. aeruginosa to persist in both community and hospital settings. This study was done to find the prevalence rate of Meropenem resistant Pseudomonas aeruginosa carrying metallobeta lactamase producing genes.

Materials and Methods: The study included a total of 100 non-duplicate clinically significant, random bacterial isolates obtained from a North Indian rural tertiary care hospital (Sri Guru Ram Das Institute of Medical Sciences and Research, Amritsar, India) from various clinical specimens, over a period of one year (January2012 to December 2012) . Identification of organisms was done using the standard microbiological techniques as described by Colle et al 1996. ${ }^{7}$ The antimicrobial susceptibility testing was performed by Kirby Bauer disc diffusion method. To detect MBL producing isolates phenotypicaly, Disc potentiation test (DPT) and Double disc synergy test (DDST) were performed. PCR was performed on Meropenem resistant isolates to detect bla IMP $_{\text {and }}$ blavIM genes.

Results: Out of 100 examined isolates, 36\% isolates were resistant to Meropenem. Out of these Meropenem resistant strains, 44.4\%(16) P. aeruginosa isolates were MBL producers as detected by DDST method and 69.44\%(25) isolates were MBL producers phenotypically by DPT method. PCR revealed prescence of blavIM gene in 16.66\%(6) of Meropenem resistant Pseudomonas aeruginosa isolates and $38.88 \%$ (14) isolates were found to be carrying bla $I_{I M P}$ gene . Three isolate were shown to carry both blaimp and blavim genes.

Conclusion: Resistance of $P$. aeruginosa isolates to Meropenem due to MBL enzymes is increasing in rural area. Resistance due to MBL has clinical significance, rapid detection of MBL producing strains followed by appropriate treatment is necessary to prevent the spreading of these organisms.
\end{abstract}

Keywords: Pseudomonas aeruginosa, PCR, Metallobetalactamase.

\section{Introduction}

Pseudomonas aeruginosa is a ubiquitous Gramnegative bacteria present in many diverse environmental settings. The wide metabolic versatility and high intrinsic and acquired resistance to many antimicrobial agents have allowed $P$. aeruginosa to persist in both community and hospital settings. It is one of the major organisms responsible for nosocomial infections such as pneumonia, UTIs, surgical site infections, and bloodstream infections. ${ }^{1}$

According to data from the Centre for Disease Control and Prevention (CDC) National Nosocomial Infections Surveillace system, Pseudomonas aeruginosa can be rated as the most common cause of ICU related pneumonia. Second most common cause of nosocomial pneumonia. This organism is third most common cause of hospital acquired UTI and fourth in order to cause surgical wound infections .It is the most common gram negative organism to be isolated from corneal ulcer $\&$ endocarditis. ${ }^{2}$

Blood stream infections caused by Pseudomonas aeruginosa have $50 \%$ higher mortality rates than other gram negative bacteremia. Mortality because of pneumonia along with pseudomonal bacteremia occurs typically after 3-4 days of appearance of signs and symptoms of infection. Mortality rates due to VAP caused by this organism are approximately $68 \%$ higher than other organisms. ${ }^{2}$

Intrinsic resistance of Pseudomonas aeruginosa to many known antibiotics as well as acquired resistance to multiple drugs leading to multi drug resistant strains (MDR) are the important reasons of high mortality rates of this organism. ${ }^{1}$

The drugs of choice against MDR Pseudomonas aeruginosa and other non fermenters are Carbapenems. ${ }^{3}$

However, Carbapenem resistance in nonfermenting bacteria such as Acinetobacter spp. and P. aeruginosa is increasing worldwide and poses a major public health threat. The mechanisms of carbapenem resistance include the production of $\beta$-lactamases, efflux pumps, and mutations altering the expression and function of porins and PBPs. ${ }^{4}$

Instead of these intrinsic $\beta$-lactamases, several other acquired $\beta$-lactamases have been identified as inducing carbapenem resistance. These acquired enzymes belong either to the class B enzymes (also 
known as metallo- $\beta$-lactamases, (MBLs) or to the class D enzymes (also known as oxacillinases).

Carbapenemases can be classified into two main molecular families: those with serine at their active site, known as serine carbapenemases, and those with at least one zinc atom at their active site known as metallo-carbapenemases, which are considered as subgroup of metallo-beta-lactamases (MBLs). The VIM, IMP and SPM types are the most clinically significant carbapenemases which encoded by blaIMP, blaVIM and blaSPM genes. ${ }^{5}$ MBLs such as VIM and IMP confer a high level of carbapenem resistance in nonlactose fermenter gram negative bacilli, as well as resistance to all $\beta$-lactams except aztreonam. ${ }^{6}$

The high resistance for antibiotics and the rapid dissemination of metallobetalactamase producing isolates in hospital require an effective phenotypic method for identifying them as there are no prescribed CLSI guidelines available for detecting them. This study was conducted to find the prevalence of MBL and their resistance genes, namely bla MDR Pseudomonas aeruginosa strains isolated from specimens received in a rural tertiary care hospital.

\section{Materials and Methods}

All the $P$. aeruginosa isolates were obtained from a North Indian rural tertiary care hospital (Sri Guru Ram Das Institute of Medical Sciences and Research, Amritsar, India) from various clinical specimens, such as endotracheal aspirates, cerebrospinal fluid, wound swabs, urine and blood culture specimens, from patients admitted to various wards from over a period of one year (January2012 to December 2012)

Identification of organisms was done using the standard microbiological techniques as described by Colle et al $1996 .{ }^{7}$ The study included a total of 100 nonduplicate clinically significant, random bacterial isolates.

The antimicrobial susceptibility testing was performed by Kirby Bauer method. Antimicrobial agents and their disc concentrations used are as follows; (HIMEDIA) Amikacin 30 $\mathrm{\mu g}$, Ciprofloxacin5 $\mu \mathrm{g}$,

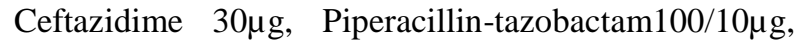
Imipenam10 $\mu \mathrm{g}, \quad$ Meropenem10 $\mu \mathrm{g}$, Polymyxin 300

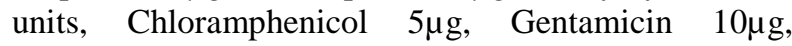
Norfloxacin $10 \mu \mathrm{g}$.

The results were interpreted as per CLSI (Clinical Laboratory Standards Institute) guidelines. ${ }^{8}$

The carbapenem resistant MDR Pseudomonas aeruginosa were further screened for MBL production. ${ }^{9}$ Minimum inhibitory concentration (MIC) of the isolates was determined by Epsilometer test (E-test) for Meropenem. E strips (AB bioMerieux, Sweden) containing predefined gradient of antibiotic were used. ${ }^{10}$

To detect MBL producing isolates Disc potentiation test (DPT) as described by Yong $\mathrm{D}$ et $\mathrm{al}^{11}$ and Double disc synergy test (DDST) as described by
Lee $\mathrm{K}$ et al. ${ }^{12}$ Combination used for DDST was MeEDTA. For DPT combination used in this test is Me $(10 \mu \mathrm{g})$ and Me-EDTA for zone enhancement. All the MDR Meropenem resistant isolates were checked genotypically by PCR for the presence of predominant genes bla $_{I M P}$ and bla $_{V I M}$. Primers for the PCR were procured from BIOSERVE (Hyderabad)

MBL GENE IDENTIFICATION

MULTIPLEX PCR FOR IMP and VIM ${ }^{13}$

Duplex PCR was performed for IMP AND VIM genes.

The primer sequence for IMP-F(5'-

3');TTGACACTCCATTTACDG

The primer sequence for IMP-R(5'-

3');GATYGAGAATTAAGCCACYCT

This amplified a 139bp amplicon.

The primer sequence for VIM- $\mathrm{F}\left(5^{\prime}-\right.$ 3');GATGGTGTTTGGTCGCATA

The primer sequence for VIM-R (5'-

3'); CGAATGCGCAGCACCAG

This amplified a $390 \mathrm{bp}$ amplicon.

The data thus obtained was tabulated, compiled and analysed.

\section{Results}

Among the hundred study isolates, maximum number of isolates were obtained from pus samples i.e 58 isolates.(Table-1).Table-2 shows the distribution of isolates from various wards. Antimicrobial sensitivity pattern shows that maximum number of isolates were resistant to Chloramphenicol i.e 69 isolates and 36 isolates were found to be resistant to Meropenem. (Table 3).

All the isolates were sensitive to polymyxin B. Meropenem resistant isolates were also found to be MDR. MIC of Meropenem by Epsilometer test revealed $29 \%$ isolates with intermediate to complete resistance. (Table 4). Comparison of resistance of isolates by disc diffusion method and E Test shows 36\% and 29\% resistance respectively.7\% isolates detected resistant in disc diffusion method were having their MIC in the sensitive range.

Out of 36 Meropenem resistant strains, 44.4\%(16) $P$. aeruginosa isolates were MBL producers as detected by DDST method and $69.44 \%(25)$ isolates were MBL producers by DPT method. (Table 5)

PCR revealed amplification of a 390bp fragment corresponding to bla $_{V I M}$ gene in $16.66 \%$ (6) isolates and $38.88 \%$ (14) isolates were found to be carrying bla IMP gene corresponding to $139 \mathrm{bp}$. (Table 6)

In our study $b l a_{I M P}$ was the predominant gene.

Three isolate were shown to carry both bla IMP $_{\text {and }}$ bla ${ }_{V I M}$ genes. 
Table 1: Distribution of $P$. Aeruginosa in indoor patients

\begin{tabular}{|l|c|}
\hline \multicolumn{1}{|c|}{ Sample } & P. Aeruginosa \\
\hline Blood & 2 \\
\hline Body Fluids & 1 \\
\hline ETT sec & 13 \\
\hline Pus & 58 \\
\hline Sputum & 8 \\
\hline Urine & 18 \\
\hline Total & 100 \\
\hline
\end{tabular}

Table 2: Source of $P$. aeruginosa isolates in various wards of institute

\begin{tabular}{|l|c|}
\hline \multicolumn{1}{|c|}{ Ward } & P.aeruginosa \\
\hline Emergency/W & 5 \\
\hline Eye/W & 2 \\
\hline Gynae/W & 1 \\
\hline ICU & 25 \\
\hline Med/W & 10 \\
\hline Ortho/W & 9 \\
\hline Paed/W & 3 \\
\hline Surgery/W & 45 \\
\hline Total & 100 \\
\hline
\end{tabular}

Table 3: Antibiotic susceptibility pattern of P. aeruginosa.

\begin{tabular}{|l|c|c|c|}
\hline Antimicrobials & \multicolumn{3}{|c|}{ P. aerugenosa } \\
\hline & Sensitive & Intermediate & Resistant \\
\hline AK & 91 & 3 & 6 \\
\hline G & 52 & 0 & 48 \\
\hline CIP & 41 & 0 & 59 \\
\hline CAZ & 38 & & 62 \\
\hline PIT & 82 & 1 & 17 \\
\hline IMP & 75 & 0 & 25 \\
\hline MP & 64 & 0 & 36 \\
\hline CHL & 13 & 0 & 69 \\
\hline NX & 7 & 0 & 11 \\
\hline P-B & 100 & 0 & 0 \\
\hline
\end{tabular}

Table 4: MIC (E test Meropenem) in P.aeruginosa isolates

\begin{tabular}{|l|c|}
\hline \multicolumn{1}{|c|}{ E Test } & P.aeruginosa \\
\hline Sensitive & 71 \\
\hline Intermediate & 1 \\
\hline Resistant & 28 \\
\hline Total & 100 \\
\hline
\end{tabular}

Table 5: Results of DDST and DPT

\begin{tabular}{|l|c|c|c|}
\hline Test & $\begin{array}{c}\text { Performed } \\
\text { on isolates }\end{array}$ & $\begin{array}{c}\text { MBL } \\
\text { producers }\end{array}$ & $\begin{array}{c}\text { NON MBL } \\
\text { producers }\end{array}$ \\
\hline DDST & 36 & $16(44.4 \%)$ & 20 \\
\hline DPT & 36 & $25(69.44 \%)$ & 11 \\
\hline
\end{tabular}

Table 6

\begin{tabular}{|l|c|c|c|c|c|}
\hline Sample & $\begin{array}{c}\text { Total Isolates } \\
\text { Obtained }\end{array}$ & $\begin{array}{c}\text { Meropenem } \\
\text { Resistant }\end{array}$ & $\begin{array}{c}\text { MBL Producers } \\
\text { by DPT Method }\end{array}$ & IMP & VIM \\
\hline PUS & 58 & 18 & 12 & 6 & 1 \\
\hline Urine & 18 & 7 & 5 & 3 & 2 \\
\hline $\begin{array}{l}\text { ET Tube } \\
\text { Secretions }\end{array}$ & 13 & 10 & 8 & 5 & 3 \\
\hline Sputum & 8 & 1 & 0 & 0 & 0 \\
\hline Blood & 2 & 0 & 0 & 0 & 0 \\
\hline Body fluids & 1 & 0 & 0 & 0 & 0 \\
\hline Total & 100 & 36 & 25 & 14 & 6 \\
\hline
\end{tabular}

\section{Discussion}

$P$. aeruginosa isolated from pus were $58 \%$, urine $18 \%$, ETT secretions $13 \%$, sputum $8 \%$, blood $2 \%$, body fluids $1 \%$. While Attal RO et al isolated $28.6 \%$ \& $22.9 \%$ P. aeruginosa in pus and urine respectively, and 
$25 \%$ in sputum, $12.1 \%$ in blood, $5 \%$ in ett secretions and $6.4 \%$ in body fluids. ${ }^{14}$

Total isolates from the surgery ward were $45 \%$, followed by ICU $25 \%$ while in a study of Attal RO et al 2010 only $9.28 \%$ isolates of $P$. aeruginosa were from ICU. ${ }^{14}$

Resistance to Meropenem in P. aeruginosa is $36 \%$ .This is in accordance with the study by Sarkar et al which reports $36.36 \%$ resistance to carbapenems, ${ }^{15}$ study by Ramakrishna $\mathrm{K}^{16}$ shows $84 \%$ resistance to Meropenem. Our study revealed resistance of $P$ .aeruginosa to Imipenem $25 \%$, while in another study $40 \%$ resistance to Imipenem was detected, ${ }^{16}$ Ceftazidime showed (62\%) resistance in this study while in a previous study by Aggarwal et al resistance to Ceftazidime was $10.35 \%$, while in the study of Shahid et al and Pitt et al resistance to Ceftazidime was $20 \%$ \& $39.6 \%$ respectively. ${ }^{17}$ Among aminoglycosides, Amikacin showed least resistance in our study (6\%). This is in accordance with the study by Aggarwal et al. ${ }^{17}$ while in the study by Yilmaz NO et al, ${ }^{18}$ it is $82 \%$. Gentamicin resistance was (48\%) in our study which is in accordance with Sarkar et al (45\%) while Yilmaz NO et al, reports much higher i.e(91\%). ${ }^{18}$ We observed (59\%) resistance to Ciprofloxacin while in the study of Sarkar et al it was slightly lower i.e (50\% ). ${ }^{15}$ and as high as $(82 \%)$ by Yilmaz NO et al. ${ }^{18}$

All the isolates were sensitive to Polymyxin B, same results were also recorded in other studies. ${ }^{15-18}$ In present study MIC of Meropenem by epsilometer test revealed that $29 \%$ of $P$.aeruginosa were moderate to complete resistant, while these results are different in a south Asian study, which recorded $86 \%$ moderate to complete resistance. ${ }^{19}$

This may be due to the fact that Meropenem might be a commonly used antibiotic for treatment of various infections in their hospital setup.

So the sensitivity pattern of epsilometer test have been found to be more discriminatory as compared to disc diffusion method as 5\% resistant isolates were also found to have their MIC in the sensitivity range .

Meropenem resistant isolates were subjected for the detection of MBL production by DDST and DPT and revealed that among P. aeruginosa isolates $44.4 \%$ were MBL producers by DDST method and $69.44 \%$ isolates of P.aeruginosa were MBL producers by DPT method. While In study by Attal RO et al both DDST \& DPT were performed and prevelance of MBL producing strains in $P$.aeruginosa was $11.4 \%$ and it was $8.5 \%$ in a study by Aggarwal et al ${ }^{17}$ while acc. to study by Yilmaz NO et al $71.05 \%$ are MBL producing by DDST. ${ }^{18}$ This upward trend seems to be due to the evolving scenario of indiscriminate antibiotic use in India and free access to purchase antibiotics over the counter. Results by DPT are more in accordance with the disc susceptibility test. Also study by Manoharan et al supported the present study regarding the results of DPT. ${ }^{20}$
All 36 Meropenem resistant isolates were further subjected to investigate predominant MBL coding gene

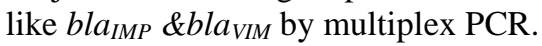

$16.66 \% P$. aeruginosa in our study harboured bla $a_{V I M}$, in contrast to $88.8 \%$ in study by Manoharan et al. ${ }^{20}$ In study by Amudhan et al, $55.5 \%$ P. aeruginosa were carrying bla $_{V I M}$ gene. Presence of bla $a_{I M P}$ gene was detected in $38.88 \%$ isolates in contrast to $3.2 \%$ by Amudhan et al. ${ }^{21}$ Of the above PCR positive isolates 3 $P$. aeruginosa isolates were showing the presence of both bla IMP $_{I M}$ and bla $_{V I M}$. Suggesting that they are coproducers of IMP and VIM genes.

The isolates that were MBL positive by DPT but negative for either bla $_{I M P}$ or bla VIM $_{\text {amplification, may }}$ have genes other than these two.

\section{Conclusion}

It can be concluded from the study that $P$. aeruginosa are leading hospital pathogens. Resistance to commonly used antibiotics is posing therapeutic challenge to the clinicians. Such strains have been implicated in many recent out breaks mostly in ICUs where extensive use of antibiotics has contributed to the selection of highly resistant strains .These strains are proficient in acquiring resistance determinants and are well accustomed to survive for prolonged periods in hospital environment.

The organisms are resistant due to production of various MBLs and by detecting genes for these antibiotic resistance will help in the study of epidemiology of these organisms and hospital infection control programme.

\section{References}

1. Lister PD, Wolter DJ \& Hanson ND. Antibacterial resistant Pseudomonas aeruginosa: clinical impact and complex regulation of chromosomally encoded resistance mechanisms. Clin Microbiol Rev .2009;22:582 -610.

2. Pseudomonas Infection: Background, Pathophysiology, Epidemiology.

3. emedicine.medscape.com/article/970904-overview

4. Gilad J, Carmeli Y. Treatment options for multidrugresistant Acinetobacter species. Drugs 2008; 68:165-89.

5. Papp-Wallace KM, Endimiani A, Taracila MA, Bonomo RA. Carbapenems: past, present, and future. Antimicrob Agents Chemother 2011;55:4943-60.

6. Nordmann P, Poirel L. Emerging carbapenemases in Gram-negative aerobes. Clin Microbiol Infect 2002;8: 321-31.

7. Moosavian M., \& Rahimzadeh M. Molecular detection of metallo- $\beta$-lactamase genes, blaIMP-1, blaVIM-2 and blaSPM-1 in imipenem resistant Pseudomonas aeruginosa isolated from clinical specimens in teaching hospitals of Ahvaz, Iran. Iran J of microbial 2015;7(1),2.

8. Mackie and McCartney. Practical Medical Microbiology vol 2. $14^{\text {th }}$ edition, New Delhi: Elseviar 2007;416,294.

9. Clinical and Laboratory Standards Institute: Performance standards for antimicrobial susceptibility testing, $16^{\text {th }}, 20^{\text {th }}$ informational supplement. CLSI document M100-S16 Wayne, PA .CLSI;2006,2010 resp . 
10. Lee H, Ko KS, Song JH, Peck KR. Antimicrobial activity of doripenem and other carbapenems against Gramnegative pathogens from Korea. Microb Drug Resist 2011;17:37-45.

11. Clinical and Laboratory Standards Institute: Performance standards for antimicrobial susceptibility testing 2011, M 100 S21CLSI;31(1).

12. Yong D, Lee K, Yum JH, Shin HB, Rossolini GB, Chong Y. Imipenem-EDTA disk method for differentiation of metallo beta lactamases producing clinical isolates of Pseudomonas spp and Acinetobacter spp. J Clin Microbiol2002; 40:3798-3801.

13. Lee K, Chong Y, Shin H B, Kim Y A, Yong D, Yum JH. Modified Hodge and EDTA disk synergy test to screen metallo beta lactamase producing strains of Pseudomonas and Acinetobacter species. Europ Society Clin Microbiol Infec 2007;7(2):88-102.

14. Dallenne C, Da Costa A, Decre D, Favier C, Ariet G. Development of a set of multiplex PCR assays for the detection of the genes encoding important $\beta$-lactamases in Enterobacteriaceae. J Antimicrob Chemother 2010;65:490-95.

15. Attal RO, Basak S, Mallick SK, Bose S.

Metallobetalactamase producing Pseudomonas aeruginosa: An emerging threat to clinicians .J Clin Diag Res2010;(4):2691-96.

16. Sarkar B, Biswas D, Prasad R. A clinico microbiological study on the Importance of Pseudomonas in nosocomially infected ICU patients with special reference to metallo- $\beta$ lactamase production. Indian J Pathol Microbiol2006; 49:44-8.

17. Ramakrishnan K, Rajagopalam S, Nair S .Molecular characterization of metallobetalactamase producing multidrug resistant Pseudomonas aeruginosa from various clinical samples. Indian J Pathol Microbiol2014;57:579-82.

18. Aggarwal G, Lodhi R B , Kamalakar U P, Khadse R K, Jalgaonkar S V ,Study of Metallobeta lactamase production in clinical isolates of Pseudomonas aeruginosa .Indian J Med Microbiol 2008;26(4):349-51.

19. Yilmaz NO et al. Prevelance and molecular characterization of metallobetalactamase producing strains of Imipenem resistant Pseudomonas aeruginosa in Turkey. Indian J Med Microbiol2014,32(3):349-50.

20. Kottahachchi J , Faoagali J , Klienschmidt S . Comparison of Meropenem MIC by Etest and VITEK 2 in resistant Pseudomonas and Acinetobacter isolates. Sri Lanka J Infec Dis 2010;1(2):28-35.

21. Manoharan A, Chatterjee S, Mathai D . Detection and characterization of metallo beta lactamases producing Pseudomonas aeruginosa. Indian J Med Microbiol 2010;28(3):241-4.

22. Amudhan M S, Sekar U, Kamalnathan A, Balaraman S . bla Imp and blavim mediated carbapenem resistance in Pseudomonas and Acinetobacter species in India .J Infect Dev Ctries $2012 ; 6(11): 757-62$. 